\title{
Intrarectal administration of hCG (Human Choriogonadotropin) and fat loss as assessed by Dual energy $X$-ray absorptiometry (DXA) in experimental animals.
}

\section{Authors:}

1. Daniel Belluscio, MD.

The Oral hCG Research Center. Guido 1953 Apartment 6 "14". ZIP code: 1119.

Buenos Aires. Argentina.

Phone number: +54 114804 9784. Fax: +54 1148049784.

hcgobesity@fibertel.com.ar

2. Ezequiel Klimovsky MD.

QUID LATAM Consulting. Corrientes Av. 1922 Apartment 7 "75". ZIP code: 1045.

Buenos Aires. Argentina.

Phone number: +5411 4952 1360. Fax: +5411 49521360.

eklimovsky@quid-consulting.com

3. Alejandro Kaniuk, Vet.

Biofucal.

http://www.fucal.com/

4. Sergio Ariel Vaney

The hCG Research Center

savaney2003@yahoo.com.ar

Abstract:

Our studies suggest that in experimental animals, submitted to a hypocaloric diet, the intrarectal administration of hCG (Human Chorionic Gonadotropin)decreases body fat and increases lean mass content in relative values to a greater extent that control animals who did not receive hCG

\section{Objective:}

To determine the modifications of DXA assessments (regarding lean mass, and body fat content) before and after the administration of four different doses of hCG $(5,10,20,40$ IU / day) administered daily intrarectal for 6 weeks in conjunction to a hypocaloric diet in experimental animals. 


\section{Design and Methods:}

For this study, 28 Sprague-Dawley rats were selected. All the animals were previously submitted to a cafeteria diet for a period of 5 weeks. After this "fattening" period all the animals reached an average of $35 \%$ of weight increase.

Thereafter, animals were submitted to a hypocaloric diet (a third of the normal intake) for a period of 44 days, with water "ad-libitum" and environmental conditions similar to control animals.

Animals were splitted in five groups:

1. Control group (0): only hypocaloric diet

2. Group 1: hypocaloric diet plus the administration of intrarectal 5 IU of hCG dissolved in a cyclodextrin solution $(16 \mathrm{mg} / \mathrm{ml}$.).

3. Group 2: hypocaloric diet plus the administration of intrarectal 10 IU of hCG dissolved in a cyclodextrin solution $(16 \mathrm{mg} / \mathrm{ml}$.). .

4. Group 3: hypocaloric diet plus the administration of intrarectal 20 IU of hCG dissolved in a cyclodextrin solution $(16 \mathrm{mg} / \mathrm{ml}$.).

5. Group 4: hypocaloric diet plus the administration of intrarectal $40 \mathrm{IU}$ of hCG dissolved in a cyclodextrin solution $(16 \mathrm{mg} / \mathrm{ml}$.).

The study lasted 6 weeks.

\section{Procedures :}

To determine the percentage body fat and lean body mass per body region, we performed Dual energy X-ray absorptiometry (DXA) on a Hologic Discovery A device, on days 0 (when starting the hypocaloric diet, after the cafeteria diet fattening period) and on day 44 (end of study). Also, during these determinations, three Regions Of Interest (ROI) were selected:

Region 1: Thorax and upper extremities

Region 2 Trunk

Region 3: Lower extremities and hips.

Personnel in charge of the study were blinded to the assignment of treatment groups. 


\section{Results:}

At the end of the study the lowest mean value of body fat loss (absolute and relative) was recorded in group 4, both in all the regions (ROI) and the net content of body fat.

In addition, group 4 was more homogeneous regarding fat loss. However, the differences were not statistically significant.

The body fat decrease (absolute and relative) by region and total was slightly higher in the animals of group 4. This group also was more homogeneous.

At the end of the study, the highest values of total body fat content (absolute and relative values) were observed in group 1, both in regions 1, 2, 3, and total. However, when comparing to the rest of the groups, the differences were not statistically very significant.

When estimating fat loss in relative values, the body fat content decreases were

1. Between $12 \%$ (Group 1 ) and $15 \%$ (Group 4 ) in region 1

2. Between $23 \%$ (Group 1) and $26 \%$ (Group 4) in region 2

3. Between $11 \%$ (Group 1 ) and $16 \%$ (Group 4) in Region 3

4. Between 17 (Group 1) and 21 (Group 4).

Group 3 had a greater decrease of body fat content (absolute values) in different regions, whereas the absolute values from Group 1 showed the lower decrease.

However, the differences were not statistically significant. At the end of the study the lean body tissue content recorded was higher in group 4, both in all regions and absolute (weight) and relative (\%) values results.

\section{Conclusion}

Given the small number of animals, no definite conclusions can be drawn. However, the results show a trend towards the decrease of body fat and an increase in lean mass in hCG treated animals as compared to control group, particularly in relative values (percentage). These difference results were dose-dependent of HCG administration.

An interesting finding of this study was that, compared to control animals who did not receive $h C G$, the intrarectal administration of $h C G$ modified both the fat content and the lean mass in hCG-treated animals, as assessed by DXA, both in absolute and relative values. 
We hypothesize that the enteral administration of hCG may be an alternative route providing results closely similar to injections, without the discomfort of an invasive procedure. 